\title{
Flight performance of Crew Escape System during pad abort condition
}

\author{
S. Somanath ${ }^{1 * *}$, S. Unnikrishnan Nair $^{2}$, T. Sivamurugan ${ }^{1}$ and S. L. N. Desikan ${ }^{1}$ \\ ${ }^{1}$ Vikram Sarabhai Space Centre, Indian Space Research Organisation, Thiruvananthapuram 695 022, India \\ ${ }^{2}$ Human Space Flight Centre, Indian Space Research Organisation, Bengaluru 560054 , India
}

As a prologue to the Gaganyaan project, ISRO successfully test fired one of the critical and essential technologies for human space flight, i.e. Crew Escape System (CES) Pad Abort Test on 5 July 2018. This is a strategically important flight as a part of various qualification tests that ensures emergency escape measure to quickly pull the Crew Module (CM) along with the astronauts to a safe distance from the launch vehicle in case of any eventuality. CES along with $C M$ weighing of 12.6 tonnes lifted-off at $07.00 \mathrm{~h}$ from the Sounding Rocket Complex Launch Pad, Sriharikota. The vehicle was taken to an altitude of $3 \mathrm{~km}$ using a specially developed solid motor with multiple reverse flow and scarfed nozzles with $10 \mathrm{~g}$ acceleration. Around 330 sensors on-board were used to measure its performance. The data obtained ensured that the adopted design philosophy and approaches such as CM reorientation, aerodynamic data with jet-on environment, mission sequences, performance of special solid motors, deceleration system, etc. were in order. This provides more confidence to progress to the next level of demonstration as a part of Gaganyaan.

Keywords: Crew Escape System, Crew Module, flight performance, pad abort.

\section{Introduction}

THE Crew Escape System (CES) based abort scenario is conceived as an emergency escape measure from lift-off to an altitude of around $70 \mathrm{~km}$ for the human space flight programme. In the case of abort due to any anomaly, CES takes away the Crew Module (CM) using specially designed solid motors in the vehicle. This vehicle is highly accelerated to ensure adequate safe distance from the parent launch vehicle so as to separate CM from CES. After separation, $\mathrm{CM}$ is re-oriented, enters into the atmosphere, and finally splashes down into the sea with the help of a parachute system. In this context, this article addresses overall prelude to vehicle configuration, mission objectives as well as its constraints, wind implications on mission, propulsion, aerodynamics, structures, deceleration system, new technology developed and flight performance.

*For correspondence. (e-mail: s_somanath@vssc.gov.in)

\section{Vehicle configuration - CES}

CES-pad abort configuration has a combination of spherical cone, cylinder, flare and shroud geometry. Vehicle forward structure has a length of $8 \mathrm{~m}$, which houses the CES Jettison Motors (CJMs), Pitch Motor (PM) and Low altitude Escape Motors (LEMs). The four reverse-flow nozzles of LEMs are located at the middle of the forward structure. Vehicle forward structure is followed by a conical shroud and cylinder, so-called CM Fairing (CMF). The overall height, diameter and half cone angle of CMF are $5.4 \mathrm{~m}, 3.7 \mathrm{~m}$ and $30^{\circ}$ respectively, that houses $\mathrm{CM}$ and High altitude Escape Motor (HEM) with scarf nozzle flushed with external surface. The aft portion of CES is attached with cruciform grid fins to ensure sufficient static stability under jet-on and jet-off phases. The vehicle liftoff mass is 13 tonnes and the total length is $14 \mathrm{~m}$. Figure 1 shows CES vehicle configuration.

\section{Crew module configuration}

CM has the shape of an inverted cup cake (Figure 2) with a large diameter in the forward region to minimize heat load and provide sufficient internal volume $\left(4.5 \mathrm{~m}^{3}\right)$. This is required to accommodate control system, parachute system, avionics system and the crew. The mass of CM is 4 tonnes which is designed for an impact load due to splash-down in water.

\section{Mission objectives and challenges}

During launch pad abort scenario, CES pulls CM to an altitude of approximately $2.5 \mathrm{~km}$. This is an essential requirement for normal operation of the parachute system. On the other hand, CM impact down range should be greater than $400 \mathrm{~m}$ for its recovery operations. The following are the mission objectives:

- Demonstration of abort capability at launch pad due to anomalies developed in launch vehicle.

- Demonstration of sufficient static margin with multiple jets and without jet phases.

- Demonstration of self re-orientation feature of CM.

- Demonstration of the parachute systems. 

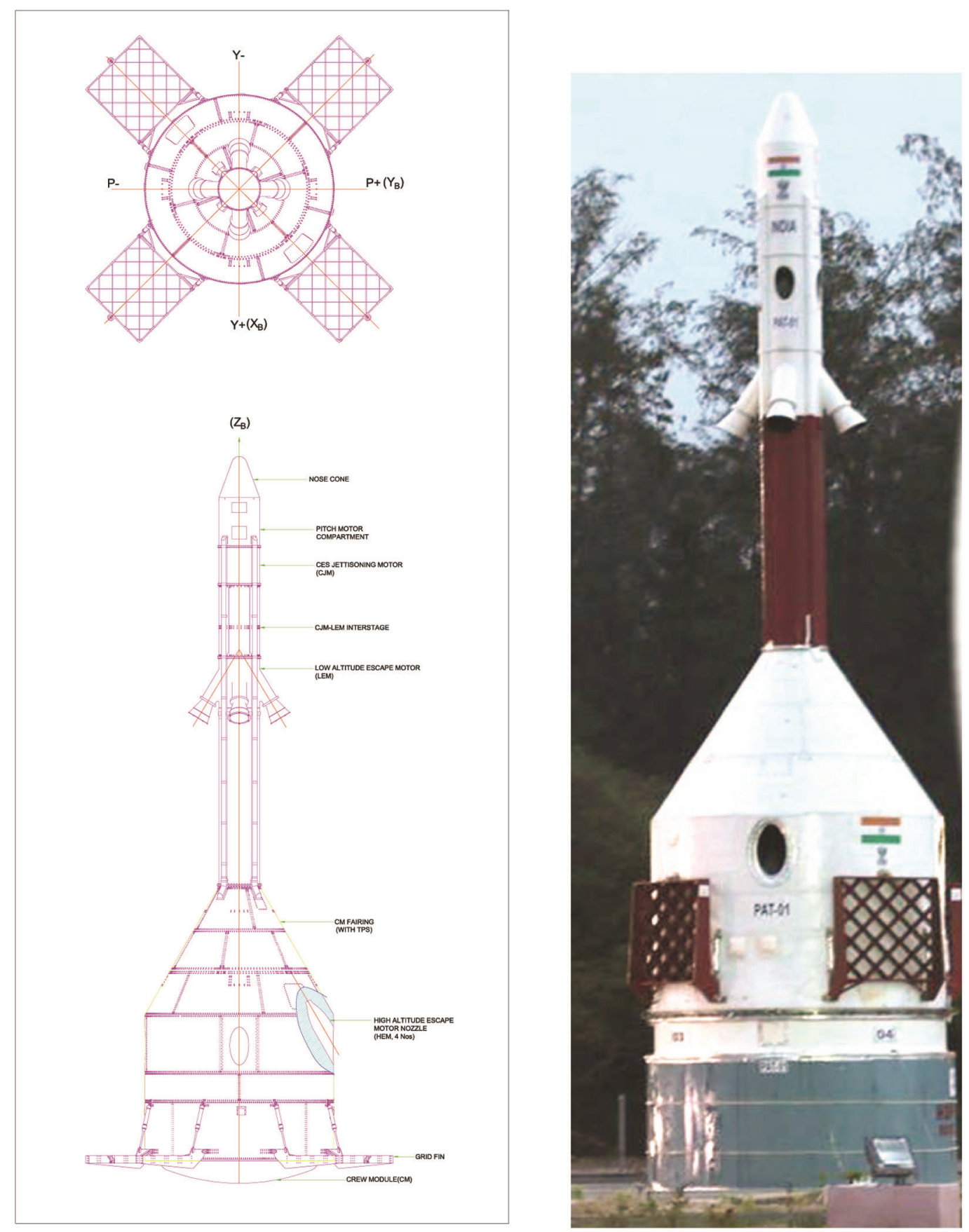

Figure 1. Crew Escape System pad abort configuration.

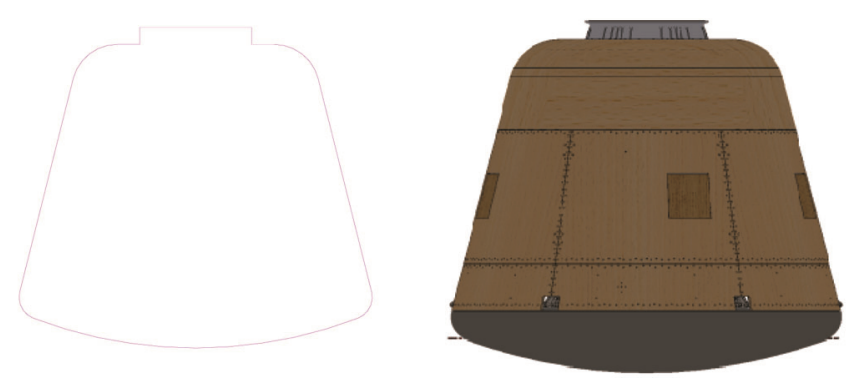

Figure 2. Crew Module configuration. 

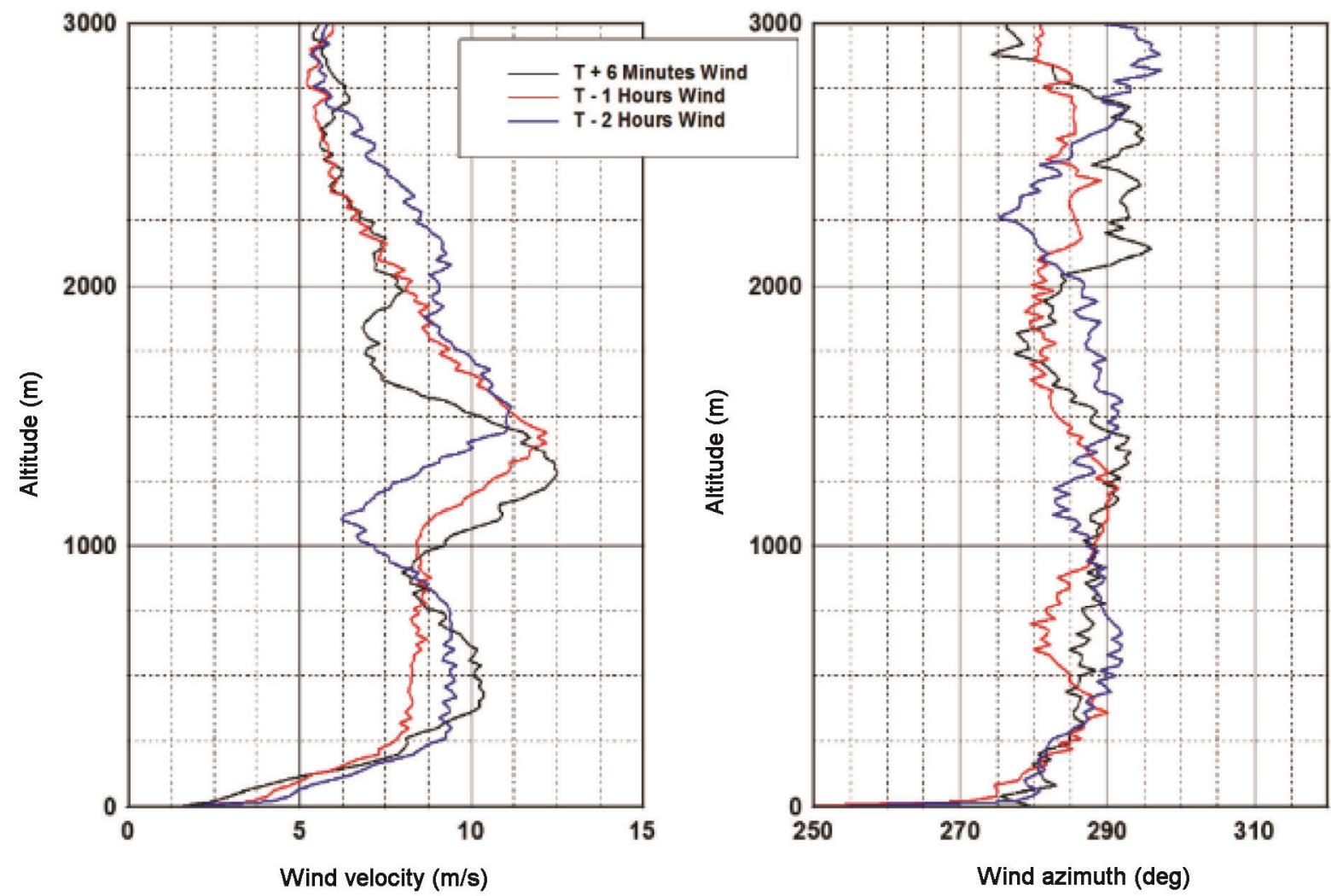

Figure 3. Wind velocity and azimuth versus altitude during launch.
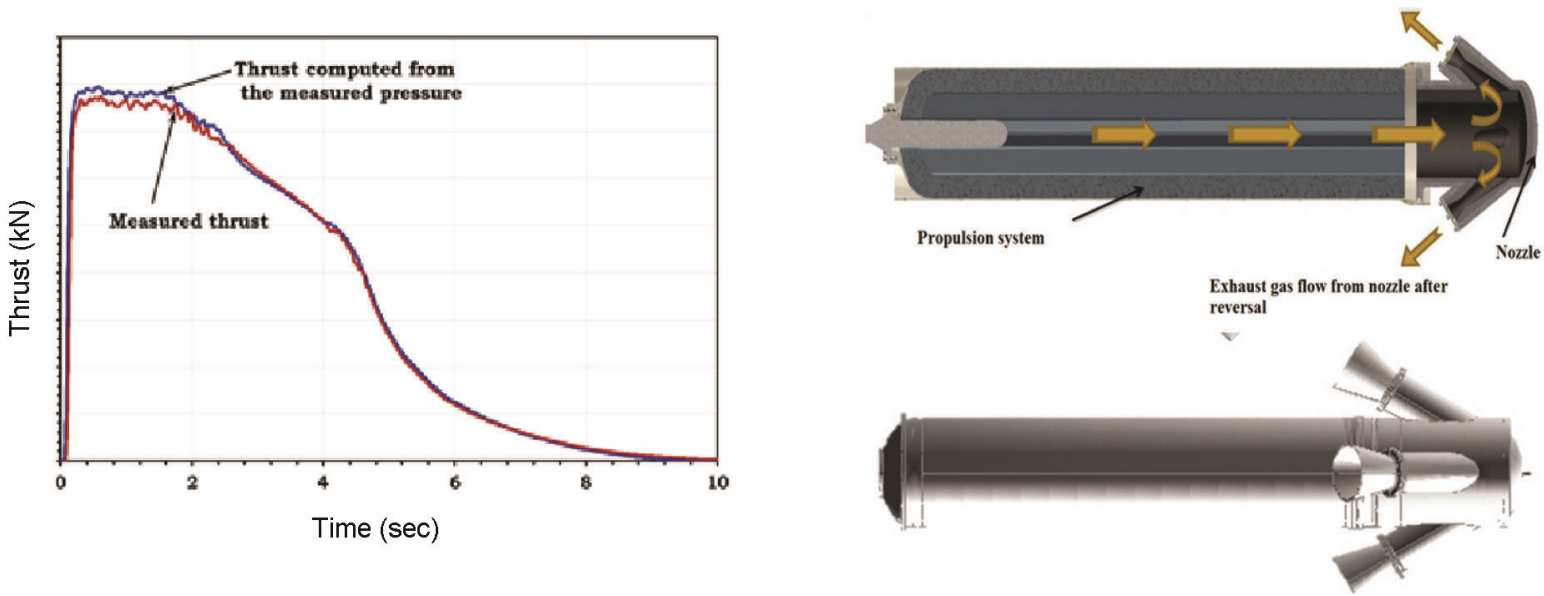

Figure 4. LEM motors and performance comparison between prediction and flight results.

The mission challenges are ensuring impact range of $\mathrm{CM}$ to be greater than $400 \mathrm{~m}$ from the launch pad and to achieve $40^{\circ}$ angle for parachute deployment. In addition, CM oscillations should be minimized in order to maintain trimmed condition. Hence sequencing of PM was studied in detail through simulations to arrive at optimum ignition time for escape motors meeting all the constraints, and the same was implemented in on-board sequence.

\section{Wind implications on mission}

The mission requirements necessitate that at the time of parachute deployment, the 'down range' should be greater than $400 \mathrm{~m}$ and the angle of attack between $\pm 40^{\circ}$. Hence, at the time of CES-CM separation, the wind induce body rates to the vehicle that play a vital role in achieving angle of attack at parachute deployment. Higher the wind speed, more the angle during parachute deployment, 
which is an unfavourable condition. In the present mission, the launch clearance was based on $6 \mathrm{D}$ simulation studies with deterministic cases (thrust performance $\pm 3 \sigma$ and all other parameters $\pm 1 \sigma$ ), and probabilistic studies with $\pm 3 \sigma$ dispersions were carried out with pre-launch winds, ensuring that constraints are not violated. However, wind measurements were carried out at different time intervals, namely $\mathrm{T} 0-1 \mathrm{~h}, \mathrm{~T} 0-2 \mathrm{~h}$ and $\mathrm{T} 0+6 \mathrm{~min}$. Figure 3 shows wind velocity and azimuth during launch. The maximum wind velocity observed was $12 \mathrm{~m} / \mathrm{s}$ and wind azimuth showed favourable conditions. Wind velocity difference of $4.5 \mathrm{~m} / \mathrm{s}$ at $1.2 \mathrm{~km}$ altitude and wind azimuth difference of $17^{\circ}$ at $2.2 \mathrm{~km}$ altitude were observed. The wind speed at $\mathrm{T} 0-2 \mathrm{~h}$ was used for probabilistic $6 \mathrm{D}$ simulation studies with 400 cases. Results indicated that all the mission constraints were understood and maximum CM impact down range was expected to be around $1.9 \mathrm{~km}$ and touch-down time of $245 \mathrm{sec}$.

\section{Propulsion performance}

CES consists of four high performance solid motors, namely LEM, HEM, PM and CJM. LEM is attached to the forward structure and the four-scarfed HEM is housed inside CMF at an angle of $31^{\circ}$ to the vehicle axis. LEM is one of the biggest motors among the four solid motors in the Pad Abort Test (PAT) vehicle whose function is to accelerate the vehicle with CM. On the other hand, in high-altitude abort case, HEM alone is used to fire. In the case of pad abort scenario, CES vehicle attains maximum acceleration to ensure crew safety, which is achieved by simultaneous firing of both LEM and HEM. These motors are newly developed with high burning rate propellant based on hydroxyl-terminated poly-butadiene/ammonium per chlorate/aluminium. The high burning rate is achieved by a coarse to fine ratio of the ammonium per chlorate particles along with two burning rate catalysts, copper chromite and ferric oxide. In LEM design, sharp

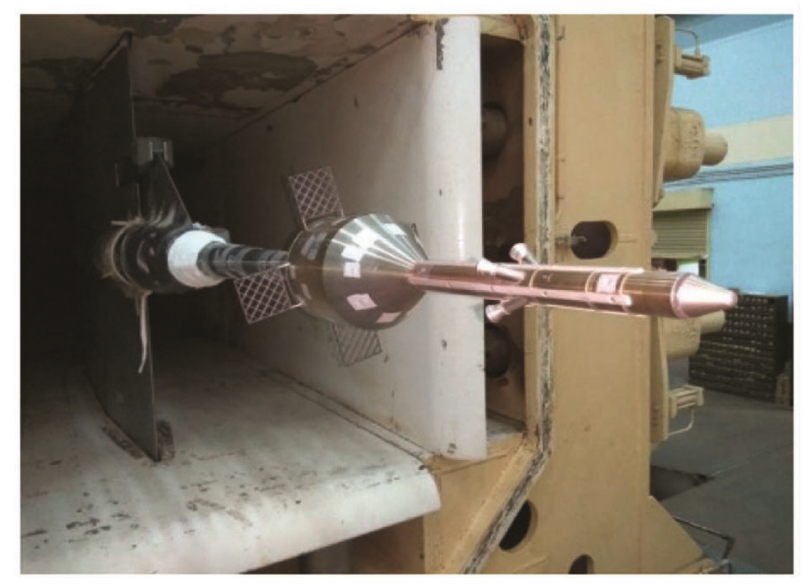

Figure 5. CES vehicle in the wind tunnel. edges and corners are avoided to make the smooth turning of internal flow with specified cant angle from thermal as well as structural point of view. Since four ellipsoidal-shaped cut-outs from the chamber cause high stress concentration, welding or other methods are not preferable. The entire LEM was machined on 15CDV6 steel forging with high precision. The solid rocket motor with four reverse-flow nozzles was newly designed, realized and its performance was evaluated from the static test and repeat tests were also carried out prior to the PAT flight. Similarly, the pressure measurements were done on the flight and the results were found to match with the pre-flight prediction (Figure 4). Tail-off was almost in line with the pre-flight trend, which revealed that the flow separation effect in these motors is also well captured.

\section{Aerodynamic coefficients}

The CES vehicle was configured to be aerodynamically stable and no active control system was used during thrusting and coasting phases of the flight. During the powered phase, three-fourth of the vehicle was engulfed with solid motor plumes which resulted in destabilizing moments. Hence, four grid fins were attached at the base of the vehicle to provide sufficient static stability from subsonic to supersonic regimes. Aerodynamic characterization of the vehicle without jet was carried out in the wind tunnel, whereas jet effects were simulated using numerical simulations (Figures 5 and 6), where the incremental effect was obtained.

\section{Module fairing and grid fin structure}

CMF has a cone-cylinder configuration and the conical panels provide mounting interface for four HEMs as well as the CM-CES truss structure which holds the CM. The aft end of the cylindrical region provides interfaces for
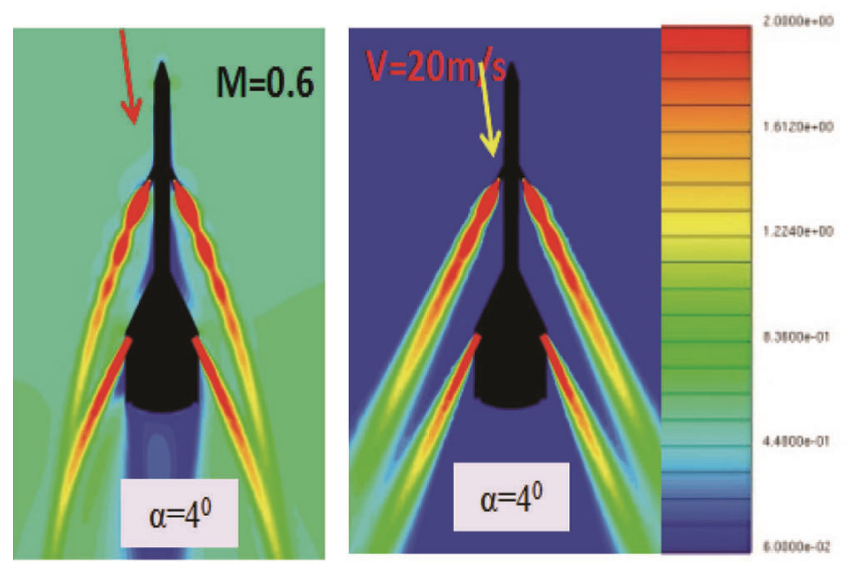

Figure 6. Mach contour of CES vehicle crew. 
grid fins along with deployment mechanisms. CMF design should cater to external pressure load, concentrated loads due to attachment of four HEMs. The fore end ring of the CMF structure interfaces the LEM. The design of the structure was done considering the entire critical load which is encountered during flight. The conical panels are proposed as iso-grid construction with improved stiffness, and ortho-grid cylindrical shells are proposed with required reinforcements for grid fin attachments. Adequacy of the end rings and bulkheads was assessed for external pressure, bending moment and loads due to local attachments. Based on Merman band tension loads, the aft end ring configuration was finalized. The grid fins are held in stowed condition during nominal mission and are deployed by pyro thrusters (Figure 7). These pyro thrusters are connected to the forward cylindrical panel through an interface flange using fasteners. These interfaces are subjected to deployment loads which are transient in nature and reinforced adequately to withstand the loads. Figure 8 shows the test scheme, set-up and instrumentation of CMF. The structural qualification was carried out for design loads and positive margin ensured prior to flight. Post-flight analysis of strain data indicate the health of the integrity of CMF during the course of the mission.

\section{Navigation}

A High Dynamic Inertial Navigation System (HDINS) was deployed in CM to support high angular rates. The sensor contains tri-axial gyros for rate measurements and three ceramic servo accelerometers for acceleration measurement. The navigation interface processor module based on Vikram 1601 was used for processing the electronic signals. The functional requirements of HDINS are CM impact sensing based on incremental velocity, $\Delta v$ from acceleration channels, measurement of rates and acceleration.

\section{Deceleration system}

The deceleration system has been designed for 4 tonne $\mathrm{CM}$ and caters to the requirements for nominal as well as abort missions. The deceleration system consists of eight parachutes and 15 pyro devices, in addition to other hardware for stowing and connecting the parachutes and their risers. The elements of the deceleration system are housed in the parachute compartment located at the aft end of CM. The parachute compartment is divided into six sectors. The system elements are located in the compartments in such a way that safe and entanglement-free extraction and deployment of parachutes are assured. The entire parachute compartment is covered by an apex cover, which is made of carbon fibre reinforced composite structures. The apex cover is ejected at the time of deceleration system initiation using three pyro thrusters. There are two parachutes used in the apex cover to prevent recontact with $\mathrm{CM}$ due to the reverse airflow. The system consisting of two identical parachute chains, each consisting of one pilot, one drogue and one main parachute. Hence the system is designed to meet the functional requirement with single chain, and the second chain is redundant to improve reliability. In addition, one-stage reefing is provided in the main parachute. The module is decelerated in three stages, viz. descent under drogue parachute, descent under main parachute and fullyopened stage. Figure 9 shows the various phases of parachute deployment.

\section{Technology development}

\section{Wireless instrumentation system}

A proof-of-concept for an IEEE802.15.4-based wireless sensor network (WSN) for aerospace applications is implemented in the flight experiment. Based on pre-flight and actual flight experimental results, wireless instrumentation scheme for the harsh aerospace environment is demonstrated. A custom static micro slot structured beaconing MAC protocol compatible with the industry popular IEEE802.15.4 PHY and amenable for diversity techniques is used to enhance communication robustness. The proposed system enables reliable communication and

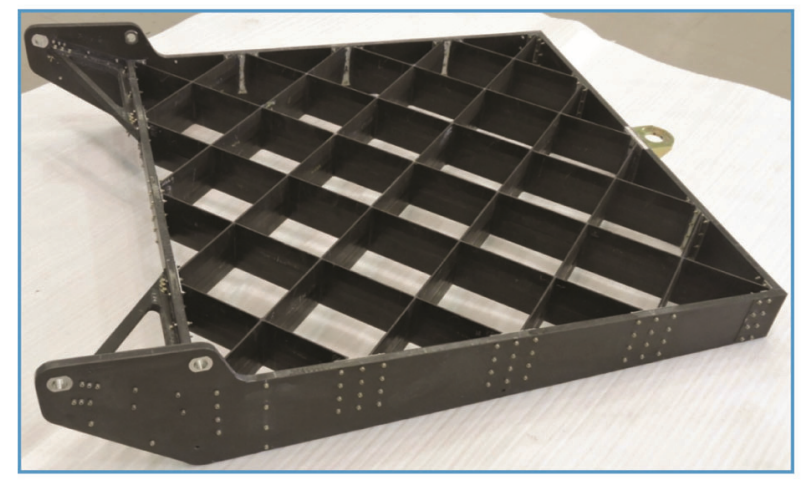

Figure 7. Grid fin structure.

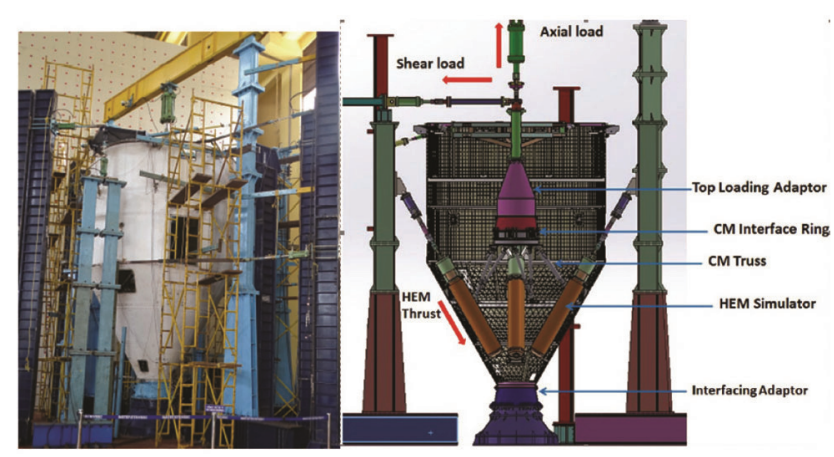

Figure 8. Structural test set-up for Crew Module Fairing. 


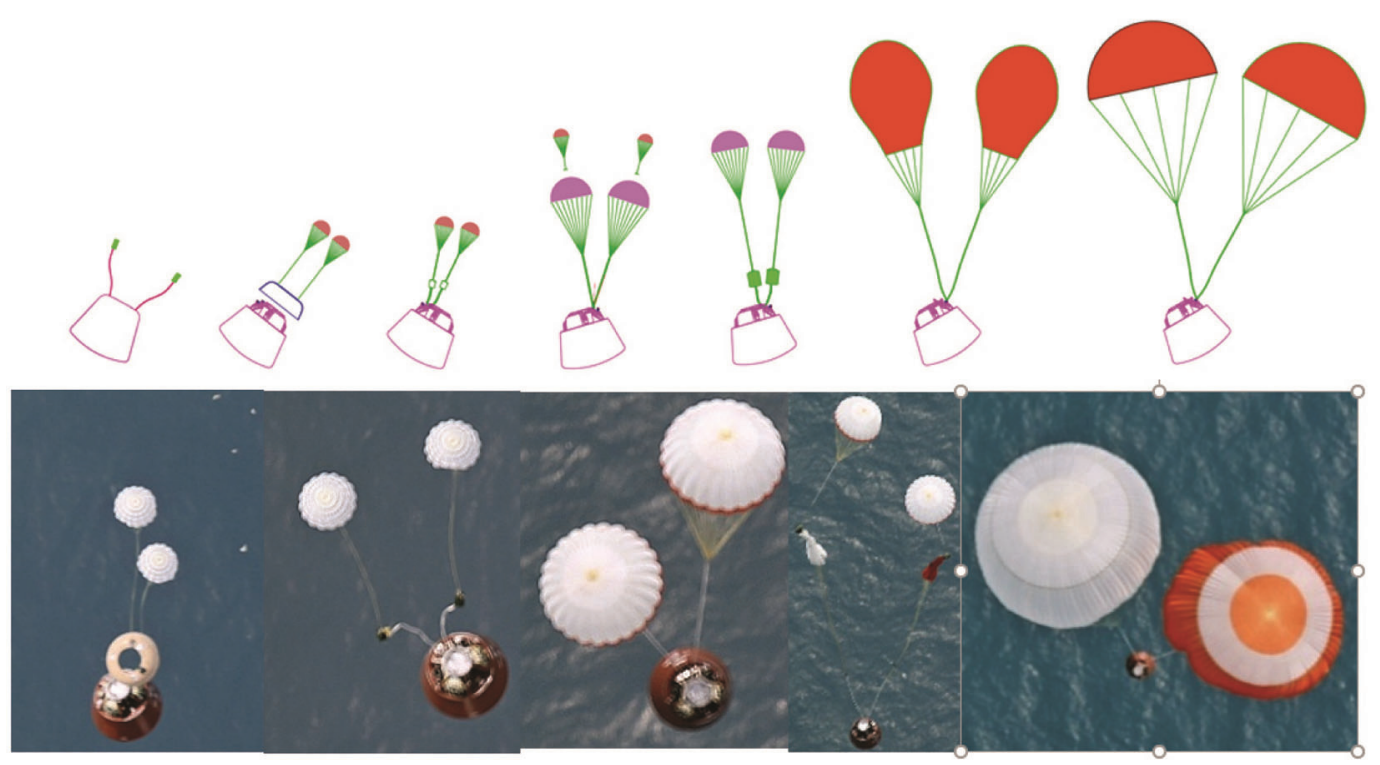

Figure 9. Various phases of parachute deployment.

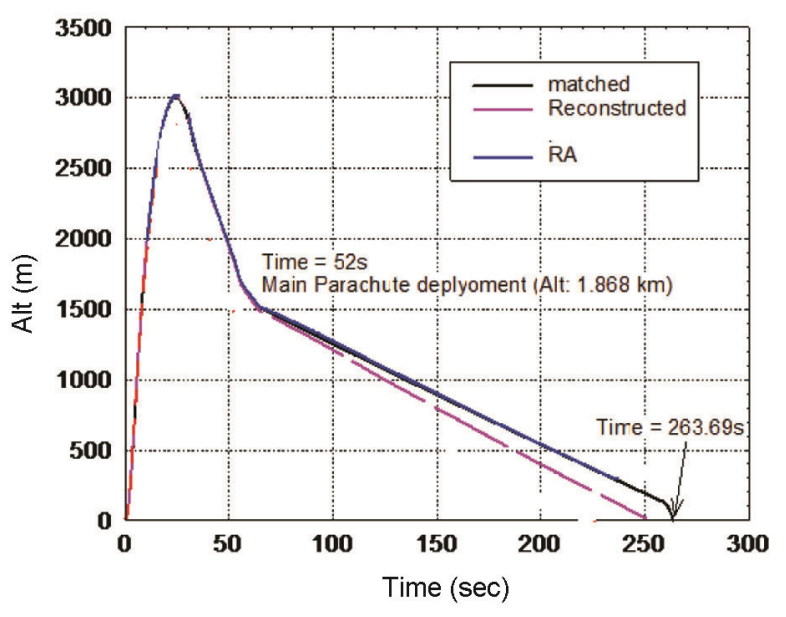

Figure 10. Mission profile of Pad Abort Test (PAT).

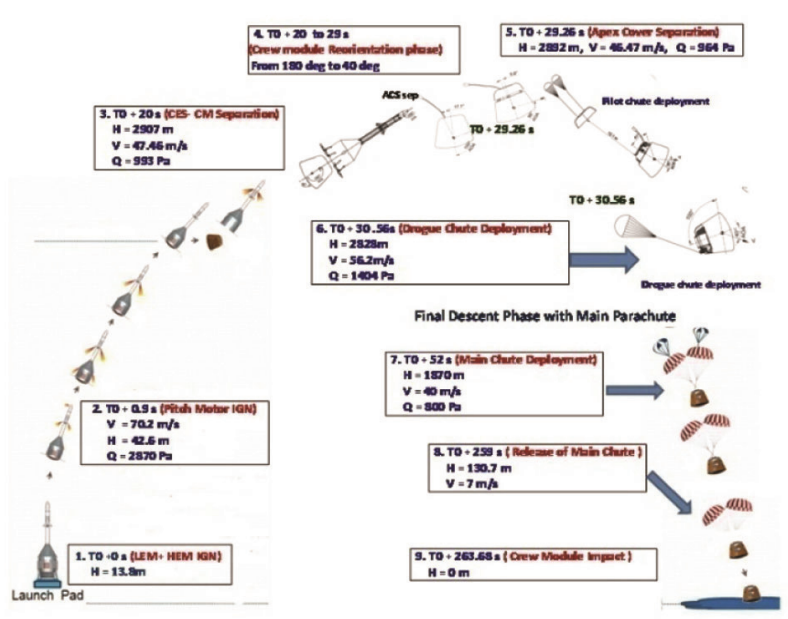

Figure 11. Altitude history during PAT mission. better bandwidth utilization without negative impact on latency or complexity of WSN. Measurements in a highly dynamic flight environment as well as simulations and performance evaluation in terms of packet error rates show that the system performs better than state-of-the-art sensor networks under a wide range of boundary conditions, especially in a closed metallic environment. Though the design can tolerate a synchronization time slip of $\pm 22 \mu \mathrm{s}$, actual time slip observed is only $\pm 6 \mu \mathrm{s}$ under extreme temperature conditions using commercial off the shelf (COTS) components. The system is scalable regarding the number of WSNs and the sensors in a single WSN. In addition to robust communication, miniaturized packing of WSN with radio frequency, in-built MEMS-based sensors and external signal conditioning are demonstrated. The use of flash-based reprogrammable Field Programmable Gate Array (FPGA) for flight applications is also proven and such FPGAs can be used for other applications as well. This flight experiment indicates that IEEE802.15.4 with a robust MAC is a suitable technology in the aerospace environment, although further experimental studies are necessary to assess the reliability and quality of service demands for aerospace applications.

\section{Digital telemetry transmitter}

A Digital Telemetry Transmitter with OQPSK direct RF modulation with turbo coding is realized and incorporated in CES. The OQPSK modulation enables the system to use less linear amplifier with minimum spectral occupancy and inter-symbol interference. Direct RF modulation offers a simple compact system with minimum component count and better reliability. Coding 
gain offered by turbo coding in the baseband system reduces the high RF transmit power requirement for establishing link for a particular data rate. With the above features, the system performed excellently in this mission and provided data for the entire mission duration.

\section{Flight performance}

\section{Flight profile}

Figure 10 depicts the CES PAT-01 mission profile. The PAT vehicle lifted off with simultaneous ignition of LEM and HEM and then the pitch control motor was ignited at $\mathrm{T} 0+0.9 \mathrm{sec}$ to pitch down of the vehicle and maximize the impact range of CM. Subsequently, the CES/CM separation took place at $\mathrm{T} 0+20 \mathrm{sec}$ and $\mathrm{CM}$ self-reoriented from 180 to $40^{\circ}$, which is essential for initiating parachute deployment. The apex cover was separated using pilot chute at $\mathrm{T} 0+29.26 \mathrm{sec}$, and the drogue chute as well as the main chute were deployed at T0 $+30.56 \mathrm{sec}$ and $\mathrm{T} 0+52 \mathrm{sec}$ respectively. The $\mathrm{CM}$ velocity was

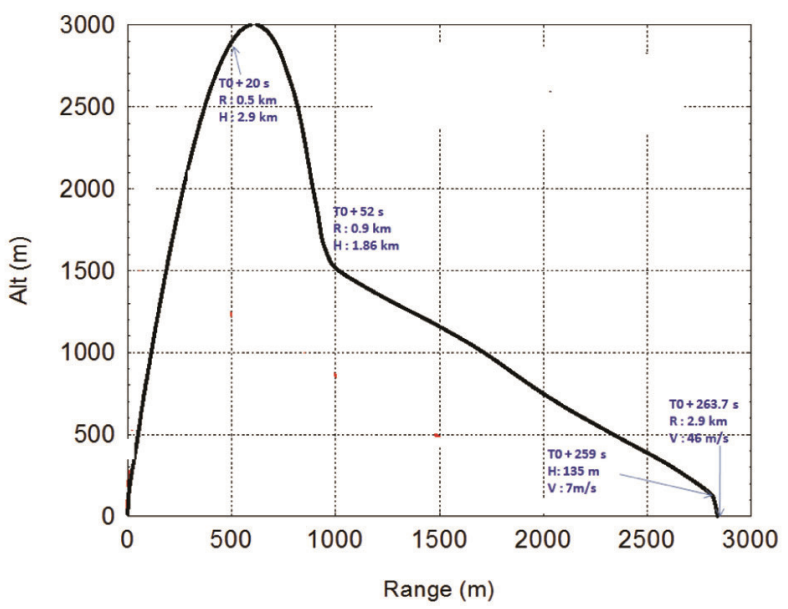

Figure 12. Velocity history - PAT.

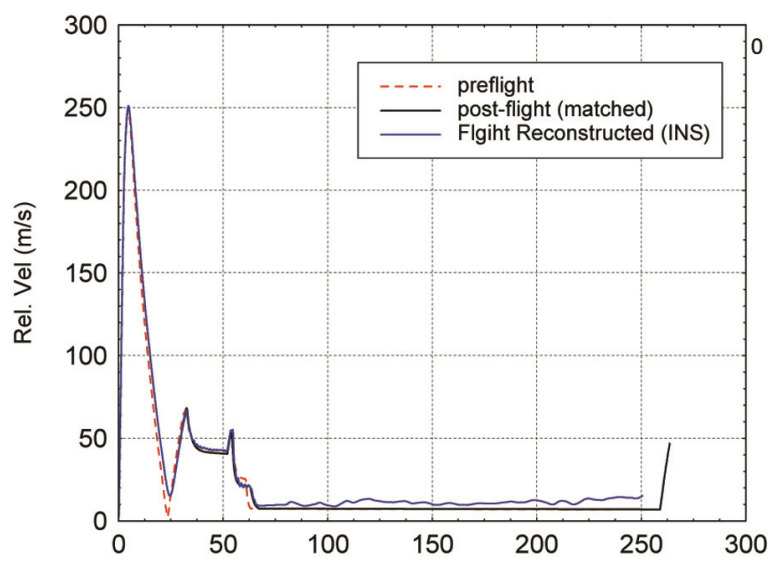

Figure 13. Altitude versus range. reduced from 56 to $7 \mathrm{~m} / \mathrm{s}$ during the parachute phase, and $\mathrm{CM}$ splashed down into the sea at $263.7 \mathrm{sec}$ (Figure 11).

Figure 12 shows the velocity profile trend. The vehicle pitched down more during PM firing phase and reached higher peak altitude compared to pre-flight, which resulted in higher down range of CM impact (Figure 13). The acceleration histories were also comparable with preflight (Figure 14). The CM total angle of attack matched with nominal aerodynamic coefficient from separation of CM onwards. As CM separated at high dynamic pressure, high angle of attack of $40^{\circ}$ was experienced during parachute deployment initiation (Figure 15).

\section{Aerodynamics}

The flight performance confirms that sufficient static margin exists in PAT vehicle from lift-off to CES vehicle separation, and that the grid fin efficiency is adequate to meet the mission requirements. The strategy adopted for

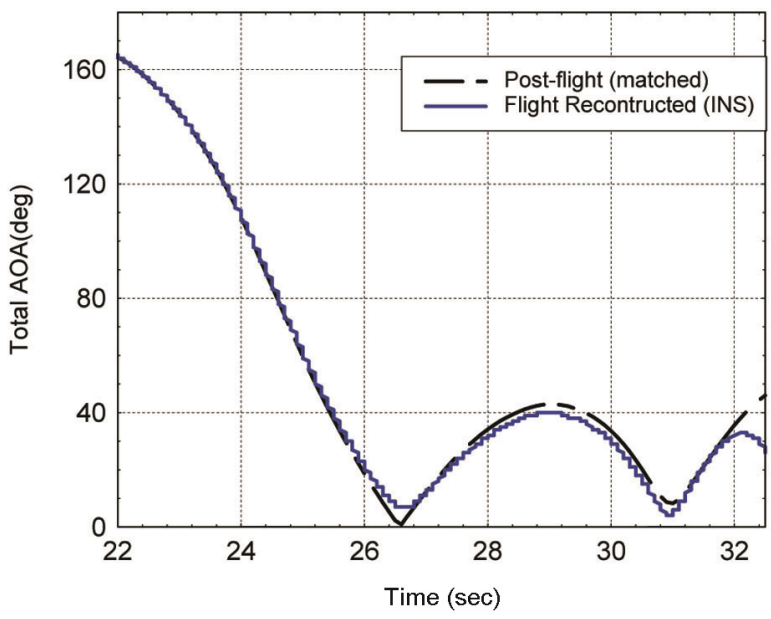

Figure 14. Acceleration history.
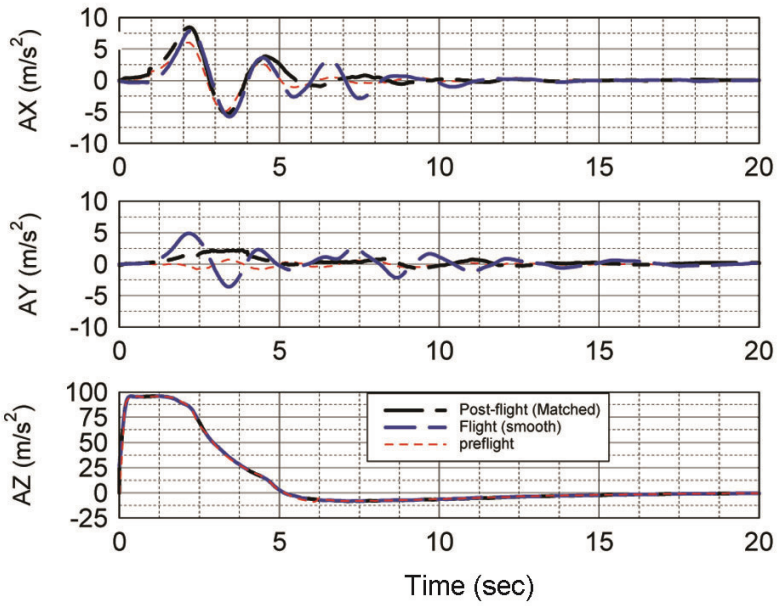

Figure 15. Angle of attack history. 


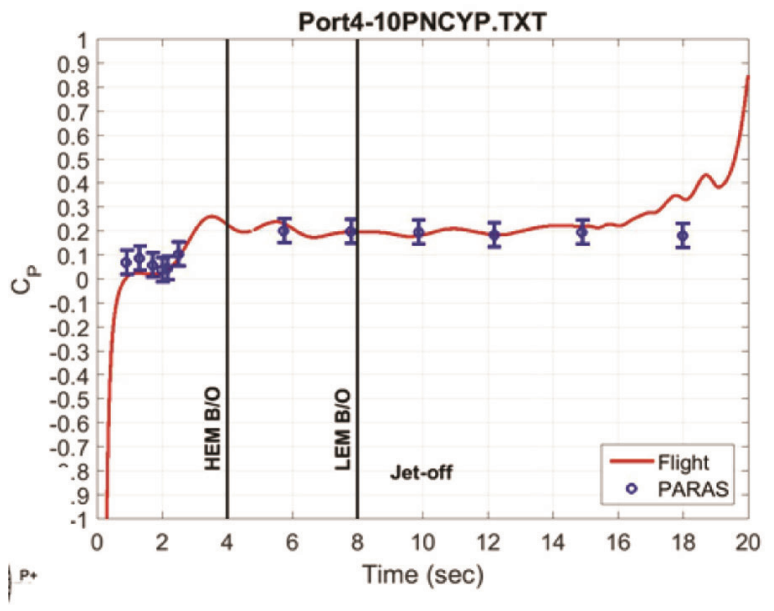

Figure 16. Pressure coefficient.

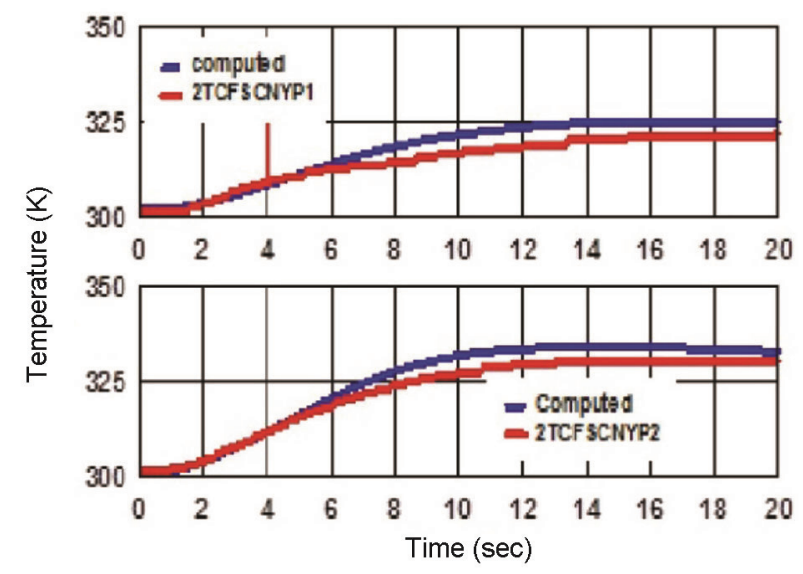

Figure 17. Measured temperature.

generating aerodynamic data under multi-jets based on numerical simulations is satisfactory. The CM aero coefficients measured using experiments with realistic external surface characteristics are also satisfactory. The pressure sensors are mounted on the flight configuration of CES and CM to assess the aerodynamic behaviour under multi jets (Figure 16). The estimated pressure coefficient from numerical simulations compared well with the measured pressure during flight.

\section{Aerothermodynamics}

The exhaust plumes from LEM and HEM are sources of heating because of their close proximity to the vehicle surface. External Thermal Protection System (TPS) designed for CMF is made of AA 2014 to protect thermal environments due to exhaust plume and maintain the structure temperature below $393 \mathrm{~K}$. Hence during flight, 12 heat flux and 20 back wall sensors were provided. It was found that all the sensors were well within the temperature constraint of $393 \mathrm{~K}$. Thermal analysis of conical region in $\mathrm{CMF}$ was carried out using heat flux sensors with implemented TPS thickness. The estimated temperature matched well with the measured temperature (Figure 17).

\section{Summary}

This article provides an overview of CES demonstration flight during Pad Abort condition. ISRO conceived CES as one of the critical technologies and prelude to the Gaganyaan project. The configuration, aerodynamics, mission planning and sequences, structural systems, parachute systems and associated sub-systems are designed, developed, integrated and successfully flight demonstrated. From these flight measurements, valuable lessons are learnt and the data indicate that the approach followed, etc. is satisfactory. Subsequent to this, it is proposed to have integrated CES flight with higher burn rate propellant motors for various abort conditions at different altitudes using tailor-made liquid stage booster. The above demonstration will validate the CES configuration performance at various altitudes and evaluate $\mathrm{CM}$, reaction control system and parachute system performance. Finally, CES configuration will be integrated on top of humanrated GSLV Mk III vehicle for human space flight.

ACKNOWLEDGEMENTS. We thank Dr K. Sivan (Chairman, ISRO) who spearheaded the programme as Director, VSSC. The encouragement and support towards this mission as well as the publication of detailed outcome through this special issue in Current Science are acknowledged.

doi: $10.18520 / \mathrm{cs} / \mathrm{v} 120 / \mathrm{i} 1 / 81-88$ 